\title{
Observer-Based Fault Tolerant Control for an Intensified Heat Exchanger/Reactor
}

\author{
Xue Han ${ }^{1, *}$, Rim Rammal ${ }^{1}$, Zetao Li $^{2}$, Michel Cabassud ${ }^{3}$ and Boutaib Dahhou ${ }^{1}$
}

\begin{abstract}
The intensified heat exchanger/reactor systems became very popular and interesting in the process intensification field. They combine both heat transfer and chemical reactions in one hybrid unit. Nevertheless, the supervision and the diagnosis of these systems is highly demanded in order to maintain their performance and ensure their safety. In this paper, a fault tolerant control system, based on a bank of adaptive observers with a backstepping-based control law, is employed to a new intensified heat exchanger/reactor, in order to detect, isolate and recover all possible dynamic faults.
\end{abstract}

\section{INTRODUCTION}

Process intensification (PI) [1], [2], [3] is an approach that involves developing new techniques and tools that are expected to improve manufacturing and processing by reducing the size of equipment while increasing production capacity, decreasing energy consumption and ultimately leading to less expensive and more sustainable technologies. In addition, PI is also a promising way to considerably reduce the danger or the consequences of a process failure in chemical, nuclear and oil industries. For example, in the chemical industry, the dissipation of heat from the reactor during an exothermic reaction is an important issue. The solution lies in the combination of reaction and heat exchanger in one hybrid unit. The heat-exchanger (HEX) reactors belong to this PI trend.

A particular intensified HEX reactor has been developed in the LGC laboratory (Laboratoire de Génie Chimique). Its model is represented in Figure 1. Despite the remarkable thermal and hydrodynamic performances of this type of HEX reactor [4], it can be strongly affected by different kind of faults: dynamic faults, sensor faults, etc. Therefore, developing a security scheme for the HEX reactor is highly demanded. The fault tolerant control (FTC) is one of the popular techniques that prevents any disruption due to a single point of failure, ensuring high availability and continuity of critical applications or systems. See survey papers [8], [9], [10]. In general, the FTC techniques are classified into two types [5]:

- Passive FTC (PFTC): consists of using a unique robust controller that deals with all the expected faults. This

\footnotetext{
*Corresponding author

${ }^{1}$ LAAS - CNRS, Université de Toulouse, CNRS, INSA, UPS, $31400 \quad$ Toulouse, France xue.handlas.fr (Xue Han), rim.rammalelaas.fr (Rim Rammal), boutaib.dahhoullaas. fr (Boutaib Dahhou)

${ }^{2}$ Electrical Engineering College, Guizhou University, Guiyang 550025 China ztli@gzu.edu. cn

${ }^{3}$ LGC, Université de Toulouse, CNRS/INP/UPS, 31432 Toulouse, France michel. cabassudeensiacet. fr
}

approach has limited fault tolerant capabilities, it is reliable only for the class of faults expected and taken into account in the design [6].

- Active FTC (AFTC): requires more information like fault detection, isolation and identification (FDI). The fault detection consists in detecting the abrupt change in the behavior of the system, fault isolation consists in determining the exact location of the fault, and fault identification provides information about the fault such as the amplitude. Using these information, the controller is redesigned online in order to maintain as best as possible the stability and other performances of the system [7]. Therefore, the AFTC are more flexible to deal with different types of faults.

Over the last three decades, various FDI methods have been developed. See survey papers [11], [12], [13]. These methods are based on the idea of generating a redundant signal for each process signal and then compare their behaviors. Among these methods there are the model-based FDI methods that use the model of the system to generate redundant signals. In these methods, the notion of residual signals, which is the difference between the process measurements and their redundancies, is introduced to detect and isolate the fault. For the HEX reactor system presented in Figure 1, model-based methods have priority since the mathematical model has already been investigated in [14]. This model takes into account both heat transfer and the chemical reaction.

Among the model-based FDI methods, observer-based approaches are proved to be effective and reliable [16], [17], [18]. In [19], five classical nonlinear observers, including extended Luenberger observer, high-gain observer and adaptive observer, were applied to the considered HEX reactor system, and it has been demonstrated that the adaptive observer has the fastest convergence speed and the minimum oscillation, and therefore the more suitable to develop an AFTC scheme to this system. Moreover, since the model of the system is available, a backstepping control redesign can be applied on the HEX reactor system in order to redesign the controller of the system and compensate the fault if it is present. In this paper, an adaptive observer-based FTC system with backstepping controller redesign are applied to the HEX reactor system in order to detect, isolate and compensate faults that occur on the system parameters, or in other words, dynamic faults.

In Section II the mathematical model of the HEX reactor system is presented and, in Section III, a control law of the system is designed based on the backstepping theory. 
Section IV presents the AFTC system used in order to detect, isolate and recover possible dynamic faults on the HEX reactor. The simulation results are illustrated in Section V, and finally Section VI concludes the paper and introduces some future work.

\section{MOdEling OF THE HEX REACTOR SYSTEM}

The considered HEX reactor system is composed of three process plates sandwiched between four utility plates, which are all engraved with $2 \mathrm{~mm}$ square cross-section channels. See Figure 1. Steel between channels, which acts as the heat exchange media, is called the plate wall. The reactants, $R 1$ and $R 2$ in Figure 1, are injected into the process channel, and the chemical reaction takes place here. Utility fluid (usually water) is injected into the utility plates to heat the process flow or take away the heat generated by the reaction. The subscript in and out represent the inlet and outlet fluid, respectively. Since there are 17 horizontal lines in each process plate, the system is divided into 17 identical units, each unit contains 15 channels: 3 process channels, 4 utility channels and 8 plate wall channels. Detailed information about the modeling process can be found in [14].

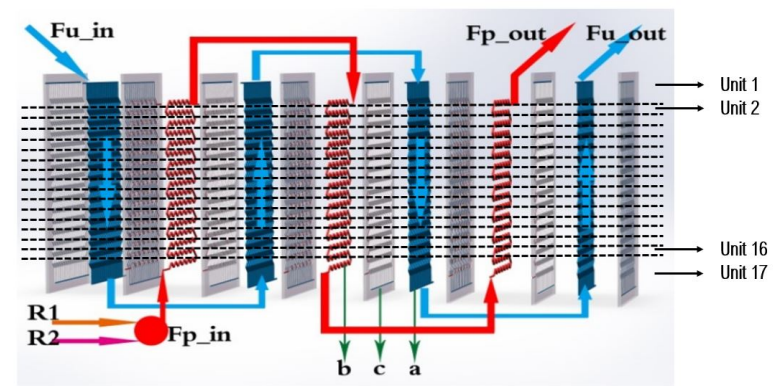

Fig. 1: HEX reactor model, (a): utility plate, $(b)$ : process plate, (c) plate wall. Source [14].

As a first step in designing an AFTC system for the considered HEX reactor, in this paper, we consider the system with only 1 unit and without a chemical reaction. The model of the system is then represented by the following equations:

$$
\begin{aligned}
& \dot{T}_{p}=\frac{F_{p}}{V_{p}}\left(T_{p, i n}-T_{p}\right)+\frac{h_{p} A_{p}}{\rho_{p} V_{p} C_{p, p}}\left(T_{w}-T_{p}\right) \\
& \dot{T}_{u}=\frac{F_{u}}{V_{u}}\left(T_{u, i n}-T_{u}\right)+\frac{h_{u} A_{u}}{\rho_{u} V_{u} C_{p, u}}\left(T_{w}-T_{u}\right) \\
& \dot{T}_{w}=\frac{h_{p} A_{p}}{\rho_{w} V_{w} C_{p, w}}\left(T_{p}-T_{w}\right)+\frac{h_{u} A_{u}}{\rho_{w} V_{w} C_{p, w}}\left(T_{u}-T_{w}\right)
\end{aligned}
$$

where $x=\left[\begin{array}{lll}T_{p} & T_{u} & T_{w}\end{array}\right]^{T}$ is the state vector, $u=$ $\left[\begin{array}{ll}F_{p} & F_{u}\end{array}\right]^{T}$ the input vector, and $y=x$ is the output vector. The variable $T$ represents the temperature, and the subscript $p, u$ and $w$ represent the process channel, utility channel and the plate wall, respectively. $F_{p}$ and $F_{u}$ are the input flow rate of process and utility fluid, respectively. The subscript in represents the inlet fluid. $\rho, V, h, A$ and $C_{p}$ are density, volume, heat transfer coefficient, heat exchange area and specific heat of material, respectively. The physical parameters of the HEX reactor system are given in Table I.

TABLE I: Physical data of the HEX reactor

\begin{tabular}{ccc}
\hline Parameter & Value & Units \\
\hline$V_{p}$ & $2.68 \times 10^{-5}$ & $\mathrm{~m}^{3}$ \\
$\rho_{p}, \rho_{u}$ & $10^{3}$ & $\mathrm{~kg} \cdot \mathrm{m}^{-3}$ \\
$C_{p, p}, C_{p, u}$ & $4.186 \times 10^{3}$ & $\mathrm{~J} \cdot \mathrm{kg}^{-1} \cdot \mathrm{K}^{-1}$ \\
$h_{p}$ & $7.5975 \times 10^{3}$ & $\mathrm{~W} \cdot \mathrm{m}^{2} \cdot \mathrm{K}^{-1}$ \\
$A_{p}$ & $2.68 \times 10^{-2}$ & $\mathrm{~m}^{2}$ \\
$V_{u}$ & $1.141 \times 10^{-4}$ & $\mathrm{~m}^{3}$ \\
$h_{u}$ & $7.5833 \times 10^{2}$ & $\mathrm{~W} \cdot \mathrm{m}^{2} \cdot \mathrm{K}^{-1}$ \\
$A_{u}$ & $4.564 \times 10^{-1}$ & $\mathrm{~m}^{2}$ \\
$V_{w}$ & $1.355 \times 10^{-3}$ & $\mathrm{~m}^{3}$ \\
$\rho_{w}$ & $8 \times 10^{3}$ & $\mathrm{~kg} \cdot \mathrm{m}^{-3}$ \\
$C_{p, w}$ & $5 \times 10^{2}$ & $\mathrm{~J} \cdot \mathrm{kg}^{-1} \cdot \mathrm{K}^{-1}$ \\
\hline
\end{tabular}

In the next section, a controller design of the system (1) (2)-(3), based on the backstepping technique, is represented.

\section{BACKSTEPPing CONTROLler Design}

For the HEX reactor system (1) - (2) - (3), we construct a control law based on the backstepping approach. The objective is to make the temperature of the process fluid $T_{p}$ follows the desired temperature $T_{p, d}$, by considering some of the states as "intermediate virtual controls". Lyapunov functions are recursively employed in order to guarantee the global stability.

In our case, since the inputs of reactants $F_{p}$ would generally have a fixed optimal proportion, the flow rate of utility fluid $F_{u}$ is set as the only input to control the temperature of the process plate $T_{p}$ : let $e_{T_{p}}$ be the process temperature tracking error between the actual value $T_{p}$ and desired temperature $T_{p, d}$ :

$$
e_{T_{p}}=T_{p, d}-T_{p}
$$

Its dynamic is given by:

$$
\begin{aligned}
\dot{e}_{T_{p}} & =\dot{T}_{p, d}-\dot{T}_{p} \\
& =\dot{T}_{p d}-\frac{F_{p}}{V_{p}}\left(T_{p, i n}-T_{p}\right)-\frac{h_{p} A_{p}}{\rho_{p} V_{p} C_{p, p}}\left(T_{w}-T_{p}\right) .
\end{aligned}
$$

Define a Lyapunov function: $V_{T_{p}}=\frac{1}{2} e_{T_{p}}^{2}$, its derivative is then given by:

$$
\begin{aligned}
\dot{V}_{T_{p}} & =e_{T_{p}} \dot{e}_{T_{p}} \\
& =e_{T_{p}}\left(\dot{T}_{p, d}-\frac{F_{p}}{V_{p}}\left(T_{p, i n}-T_{p}\right)-\frac{h_{p} A_{p}}{\rho_{p} V_{p} C_{p, p}}\left(T_{w}-T_{p}\right)\right) .
\end{aligned}
$$

In order to make $\dot{V}_{T_{p}}$ negative definite, the temperature of the plate wall $T_{w}$ is chosen as the first virtual element of control to make the tracking error $e_{T_{p}}$ converge to zero, its desired value $T_{w, d}$ is defined by:

$$
T_{w, d}=\frac{\rho_{p} V_{p} C_{p, p}}{h_{p} A_{p}}\left[\dot{T}_{p, d}+k_{1} e_{T_{p}}-\frac{F_{p}}{V_{p}}\left(T_{p, i n}-T_{p}\right)\right]+T_{p}
$$

where $k_{1}$ is a positive design parameter. By setting $T_{w}=$ $T_{w, d}$ in (6), we get $\dot{V}_{T_{p}}=-k_{1} e_{T_{p}}^{2} \leq 0$, and the stability of the tracking error system $e_{T_{p}}$ is guaranteed. 
In the next step, we define a tracking error $e_{T_{w}}$ for the plate wall temperature $T_{w}: e_{T_{w}}=T_{w, d}-T_{w}$. Similarly, we compute its dynamic:

$$
\begin{aligned}
\dot{e}_{T_{w}} & =\dot{T}_{w, d}-\dot{T}_{w} \\
& =\frac{\rho_{p} V_{p} C_{p, p}}{h_{p} A_{p}}\left(\ddot{T}_{p, d}+k_{1} \dot{e}_{T_{p}}+\frac{F_{p}}{V_{p}} \dot{T}_{p}\right)+\dot{T}_{p} \\
& -\frac{h_{p} A_{p}}{\rho_{w} V_{w} C_{p, w}}\left(T_{p}-T_{w}\right)-\frac{h_{u} A_{u}}{\rho_{w} V_{w} C_{p, w}}\left(T_{u}-T_{w}\right)
\end{aligned}
$$

and we define a Lyapunov function:

$$
V_{T_{w}}=\frac{1}{2} e_{T_{p}}^{2}+\frac{1}{2} e_{T_{w}}^{2}=V_{T_{p}}+\frac{1}{2} e_{T_{w}}^{2}
$$

with dynamic equals to:

$$
\dot{V}_{T_{w}}=\dot{V}_{T_{p}}+e_{T_{w}} \dot{e}_{T_{w}} .
$$

In order to make $\dot{V}_{T_{w}}$ negative definite, the temperature of utility fluid $T_{u}$ is chosen as the second element of virtual control to stabilize $e_{T_{w}}$, then its desired value $T_{u, d}$ is given by:

$$
\begin{aligned}
T_{u, d} & =\frac{\rho_{w} V_{w} C_{p, w}}{h_{u} A_{u}}\left[\frac{h_{p} A_{p}}{\rho_{p} V_{p} C_{p, p}} e_{T_{p}}\right. \\
& +\frac{\rho_{p} V_{p} C_{p, p}}{h_{p} A_{p}}\left(\ddot{T}_{p, d}+k_{1} \dot{e}_{T_{p}}+\frac{F_{p}}{V_{p}} \dot{T}_{p}\right)+\dot{T}_{p} \\
& \left.-\frac{h_{p} A_{p}}{\rho_{w} V_{w} C_{p, w}}\left(T_{p}-T_{w}\right)+k_{2} e_{T_{w}}\right]+T_{w}
\end{aligned}
$$

where $k_{2}$ is a positive design parameter. By setting $T_{u}=$ $T_{u, d}$ and substituting (8) in (10), we get: $\dot{V}_{T_{w}}=-k_{1} e_{T_{p}}^{2}-$ $k_{2} e_{T_{w}}^{2} \leq 0$. Finally, we define a tracking error $e_{T_{u}}$ for the utility temperature $T_{u}$ by: $e_{T_{u}}=T_{u, d}-T_{u}$ with dynamic:

$$
\dot{e}_{T_{u}}=\dot{T}_{u, d}-\dot{T}_{u} .
$$

In order to make the tracking error system $e_{T_{u}}$ converge to zero, a third Lyapunov function is used:

$$
\begin{aligned}
V_{T_{u}} & =\frac{1}{2} e_{T_{p}}^{2}+\frac{1}{2} e_{T_{w}}^{2}+\frac{1}{2} e_{T_{u}}^{2} \\
& =V_{T_{w}}+\frac{1}{2} e_{T_{u}}^{2}
\end{aligned}
$$

with a dynamic given by:

$$
\dot{V}_{T_{u}}=\dot{V}_{T_{w}}+e_{T_{u}} \dot{e}_{T_{u}} .
$$

In order to make $\dot{V}_{T_{u}}$ negative definite, we define the expression of the control law $F_{u}$ :

$$
\begin{aligned}
F_{u}= & \frac{V_{u}}{T_{u, i n}-T_{u}}\left\{\frac{h_{u} A_{u}}{\rho_{w} V_{w} C_{p, w}} e_{T_{w}}+\frac{\rho_{w} V_{w} C_{p, w}}{h_{u} A_{u}}\right. \\
& {\left[\frac{h_{p} A_{p}}{\rho_{p} V_{p} C_{p, p}} \dot{e}_{T_{p}}+\frac{\rho_{p} V_{p} C_{p, p}}{h_{p} A_{p}}\left(\dddot{T}_{p d}+k_{1} \ddot{e}_{T_{p}}+\frac{F_{p}}{V_{p}} \ddot{T}_{p}\right)\right.} \\
& \left.+\ddot{T}_{p}-\frac{h_{p} A_{p}}{\rho_{w} V_{w} C_{p, w}}\left(\dot{T}_{p}-\dot{T}_{w}\right)+k_{2} \dot{e}_{T_{w}}\right] \\
& +\frac{h_{p} A_{p}}{\rho_{w} V_{w} C_{p, w}}\left(T_{p}-T_{w}\right)+\frac{h_{u} A_{u}}{\rho_{w} V_{w} C_{p, w}}\left(T_{u}-T_{w}\right) \\
& \left.-\frac{h_{u} A_{u}}{\rho_{u} V_{u} C_{p, u}}\left(T_{w}-T_{u}\right)+k_{3} e_{T_{u}}\right\}
\end{aligned}
$$

where $k_{3}$ is a positive design parameter. Finally, by substituting (12) and (15) into (14), the dynamic of the Lyapunov function $\dot{V}_{T_{u}}$ becomes:

$$
\dot{V}_{T_{u}}=-k_{1} e_{T_{p}}^{2}-k_{2} e_{T_{w}}^{2}-k_{3} e_{T_{u}}^{2} \leq 0,
$$

hence the stability of $e_{T_{u}}$ is guaranteed.

The chemical reaction in the HEX reactor takes place in process channels, so the accumulation of products in these plates can cause them to foul. Fouling in process channels may directly influence the performance of the heat exchange between the process plates and the plate walls by decreasing the heat transfer coefficient $h_{p}$. Besides, the temperature of the inlet fluid $T_{p, i n}$ and $T_{u, i n}$ may change due to various reasons such as environmental change and malfunction of the thermocouples installed in the injection pipes. These types of faults are parameter faults or dynamic faults. In order to maintain the performance of the considered HEX reactor even in presence of a dynamic fault, an adaptive observerbased AFTC method is presented in the next section.

\section{AdAPTIVE OBSERVER-BASED AFTC}

The observer-based AFTC method is based on the mathematical model of the system. Therefore, the dynamic faulty model is needed in order to construct the AFTC scheme.

\section{A. Dynamic faulty model}

Consider the following nonlinear system:

$$
\left\{\begin{array}{l}
\dot{x}=f(x)+g(x) u+p(x) \theta \\
y=C x
\end{array}\right.
$$

where $f(x) \in \mathcal{R}^{n}$ is a vector of nonlinear functions, $g(x) \in \mathcal{R}^{n \times k}$ and $p(x) \in \mathcal{R}^{n \times m}$ are function matrices with nonlinear elements and $C \in \mathbb{R}^{q \times n}$ is a constant matrix. $x \in \mathbb{R}^{n}, u \in \mathbb{R}^{k}$ and $y \in \mathbb{R}^{q}$ represent the state vector, input vector and output vector, respectively. $\theta \in \mathbb{R}^{m}$ is the vector of system parameters. Assume that $f(x)$ and $g(x)$ are both Lipchitz.

A dynamic fault is a fault that occurs on the parameter vector $\theta$. In the following, we denote by $\theta^{0}$ the parameter vector with nominal values, by $t_{f}$ the time of occurrence of the fault, by $f_{p}$ the $m$-dimensional vector of possible parameter faults, and by $\theta_{i}^{f}, i=1, \ldots, m$, the faulty value of the $i^{\text {th }}$ parameter of $\theta$. Its expression is then given by:

$$
\theta_{i}^{f}(t)=\theta_{i}^{0}(t)+f_{p i}
$$

for $t \geqslant t_{f}$. When a fault occurs on the $i^{\text {th }}$ parameter, the nonlinear system (17) with dynamic fault can be expressed as follows:

$$
\left\{\begin{array}{l}
\dot{x}=f(x)+g(x) u+\sum_{\substack{l=1 \\
l \neq i}}^{m} p_{l}(x) \theta_{l}+p_{i}(x) \theta_{i}^{f} \\
y=C x
\end{array}\right.
$$

where $p_{l}(x)=\left[\begin{array}{lll}p_{1, l}(x) & \ldots & p_{n, l}(x)\end{array}\right]^{T}$ is the $l^{\text {th }}$ column of the matrix $p(x)$. 


\section{B. AFTC scheme based on adaptive observer}

The general structure of an AFTC system consists of a FDI method and a controller redesign mechanism. First, the fault is detected, isolated and identified, then the fault information is used for the controller redesign part to recover the fault.

In order to detect, isolate and identify the faulty parameter, we use a FDI method based on a bank of adaptive observers, proposed by the authors in [20] and [21], where each observer is specified for one faulty parameter. Then in total, we have $m$ observers corresponding to the $m$ parameters. In the following, we suppose that the system states are available, i.e. $y=x$, and that the observers are state-based observers.

For the dynamic faulty model (19), the $i^{\text {th }}$ observer, corresponding to the $i^{\text {th }}$ parameter, is constructed as follows:

$$
\left\{\begin{aligned}
\dot{\hat{x}}_{i}= & f\left(\hat{x}_{i}\right)+g\left(\hat{x}_{i}\right) u+\sum_{\substack{l=1 \\
l \neq i}}^{m} p_{l}\left(\hat{x}_{i}\right) \theta_{l}+p_{i}\left(\hat{x}_{i}\right) \hat{\theta}_{i}^{f} \\
& \quad+H_{i}\left(\hat{y}_{i}-x\right) \\
\dot{\hat{\theta}}_{i}^{f}= & -2 \gamma_{i}\left(\hat{x}_{i}-x\right)^{T} S_{i} p_{i}\left(\hat{x}_{i}\right) \\
\hat{y}_{i}= & \hat{x}_{i}
\end{aligned}\right.
$$

where $\hat{x}_{i}$ and $\hat{y}_{i}$ are the estimated state vector and output vector, respectively, $\hat{\theta}_{i}^{f}$ is the fault estimation of the $i^{\text {th }}$ faulty parameter $\theta_{i}^{f}$ and $p_{i}\left(\hat{x}_{i}\right)$ is the $i^{\text {th }}$ column of the matrix $p\left(\hat{x}_{i}\right)$. $H_{i}$ is a Hurwitz matrix that can be chosen freely in order to increase as much as possible the dynamic of the observer, $\gamma_{i}$ is a design constant, and $S_{i}$ is a positive definite matrix. $S^{i}$ can be calculated as follows:

$$
H_{i}^{T} S_{i}+S_{i} H_{i}=-Q_{i}
$$

where $Q_{i}$ is a positive definite matrix that can be chosen freely. Each observer gives an estimation of one particular parameter, and we have to choose the appropriate gain matrices $H_{i}, S_{i}$, as well as gain constant $\gamma_{i}$ to have a good fault estimation performance [20], [21].

1) Fault detection and isolation: In order to detect and isolate parameter faults, we compute a set of $m$ residual signals using the following expression:

$$
r_{k}=\left\|\hat{y}^{(k)}-y\right\|, \quad k \in 1, \ldots, m .
$$

where $\hat{y}^{(k)}$ and $y$ represent the output vector of the $k^{\text {th }}$ observer and the output vector of the system, respectively. If, at some point of the system operation, the residuals become different from zero, then a fault on the system is detected. However, if the $i^{\text {th }}$ residual converges to zero and the other $m-1$ residuals converge to a nonzero value, then the fault is isolated on the $i^{\text {th }}$ parameter. For more details see [20].

Remark 1: In the closed-loop system, the residuals can be easily affected by the change of the input signal, which makes it difficult to identify the reason for the residual change. In this paper, we suppose that all the actuators are fault-free, which eliminates the possibility that the affected residual is caused by an actuator fault.

In order to identify whether the cause of the change in the residuals is due to a change in the input signal or a fault on a system parameter, auxiliary residuals, denoted by $D r_{i}$, are introduced:

$$
D r_{i}=\frac{d\left\|\hat{y}^{(i)}-y\right\|}{d t}, \quad i \in 1, \ldots, m .
$$

These auxiliary residuals are used to evaluate the stability of the original residuals $r_{i}$. First, we check the value of the original residuals $r_{i}$ and auxiliary residuals $D r_{i}, i=$ $1, \ldots, m$, if they all leave zero then a change is detected. Second, when the auxiliary residuals $D r_{i}$ go back to zero, i.e. the original residuals are stable, we evaluate their values. If all the original residuals go back to zero, then the change is caused by an input adjustment. However, if, for example, the $i^{\text {th }}$ residual goes back to zero while the others stay at a non zero value, then the change is caused by a fault on the $i^{\text {th }}$ parameter.

2) Fault identification and recovery: Once the fault on the $i^{\text {th }}$ parameter is detected and isolated, we proceed to the calculation of the faulty value $\hat{f}_{p i}$, using the parameter estimation of the corresponding adaptive observer:

$$
\hat{f}_{p i}=\hat{\theta}_{i}^{f}-\theta_{i}^{0}
$$

In order to recover the dynamic fault, the estimated faulty value $\hat{f}_{p i}$ is used to redesign the control signal $F_{u}$ given by (15), by replacing the faulty parameter $\theta_{i}^{f}$ by $\theta_{i}^{0}+\hat{f}_{p i}$.

\section{Simulation RESUlts}

In order to validate the effectiveness of the AFTC system, illustrated in the previous section, simulations were performed using the MATLAB software environment. The objective is to make the measured process fluid temperature $T_{p}$ follows the desired value $T_{p, d}$ in presence of dynamic faults. The process fluid is injected into the process channel with a constant flow rate of $10 \mathrm{~L} \cdot \mathrm{h}^{-1}$ and a constant temperature of $77^{\circ} \mathrm{C}$, while the utility fluid is injected into the utility channel with an initial flow rate of $62.2 \mathrm{~L} \cdot \mathrm{h}^{-1}$ and a fixed temperature of $15.6^{\circ} \mathrm{C}$. The flow rates of both fluids have a range from 0 to $150 \mathrm{~L} \cdot \mathrm{h}^{-1}$ because of the physical limitation of the pumps. The initial temperatures of process channel, utility channel and plate wall are $\left[\begin{array}{lll}T_{p} & T_{u} & T_{w}\end{array}\right]^{T}=$ $\left[\begin{array}{lll}77 & 15.6 & 15.6\end{array}\right]^{T}$, respectively.

Figure 2 shows the measured temperature of the process fluid $T_{p}$ and the variable control input $F_{u}$ in the fault free case. The desired temperature $T_{p, d}$ is firstly settled at $27^{\circ} \mathrm{C}$ and then resettled at $25^{\circ} \mathrm{C}$ at $t=400 \mathrm{~s}$. To get a smoothing input signal, a filter is applied to the reference temperature. During dynamic phases (start-up and change of set-point), according to the backstepping algorithm and the physical limitation of the pump, the flow rate of utility fluid $F_{u}$ is set either to the minimum value $0 \mathrm{~L} \cdot \mathrm{h}^{-1}$ or the maximum value $150 \mathrm{~L} \cdot \mathrm{h}^{-1}$ to track the desired temperature as fast as possible.

In this paper, we consider the following dynamic faults: the heat transfer coefficient between the process plate and the plate wall $h_{p}$, which is caused by the fouling in the process channels, and the inlet temperature of the process fluid $T_{p, i n}$, 


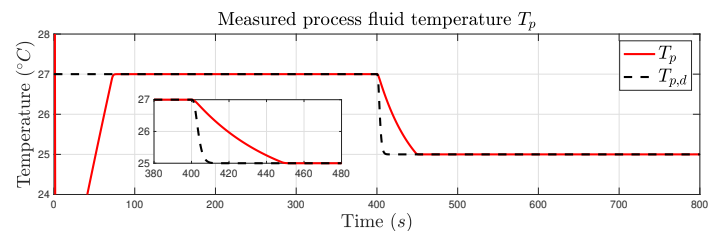

Flow rate of utility fluid $F_{u}$

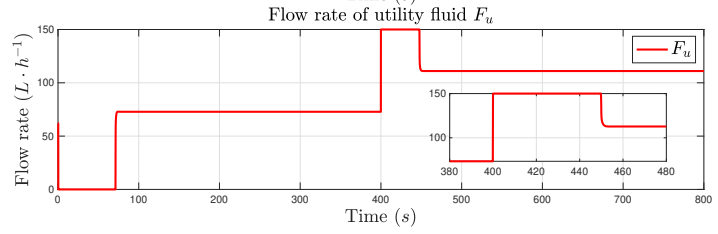

Fig. 2: Measured process fluid temperature $T_{p}$ and utility fluid flow rate $F_{u}$ in the fault free case.

which is caused by the environment temperature change or malfunction of the thermocouple in the inlet pipes. Therefore, we have two adaptive observers and hence two residuals: $r_{1}$, associated to the parameter $h_{p}$, and $r_{2}$, associated to the parameter $T_{p, i n}$.

\section{A. Dynamic fault on $h_{p}$}

At $t_{f}=200 \mathrm{~s}$, the heat transfer coefficient $h_{p}$ decreases $15 \%$ of its nominal value, i.e. $f_{p 1}=-1.393 \times 10^{3}$ $\mathrm{W} \cdot \mathrm{m}^{2} \cdot \mathrm{K}^{-1}$. Then, the heat transfer coefficient in the faulty case becomes $h_{p}^{f}=h_{p}+f_{p 1}=6.4852 \times 10^{3} \mathrm{~W} \cdot \mathrm{m}^{2} \cdot \mathrm{K}^{-1}$.

The original residuals $r_{i}$ and the auxiliary residuals $D r_{i}$, $i=1,2$, used for the FDI, are represented in Figure 3 . At about $t=72 \mathrm{~s}$, the original residuals and the auxiliary residuals change. When the auxiliary residuals go back to zero, i.e. the original residuals are stable, the original residuals also go back to zero. Then, this change is caused by a simple change of the control signal.

At $200 \mathrm{~s}$, both original and auxiliary residuals change. When the auxiliary residuals go back to zero, the original residual $r_{1}$ relative to $h_{p}$, which corresponds to the first observer goes back to zero, while $r_{2}$ stays at a nonzero value. Therefore, the fault is detected and isolated on the parameter $h_{p}$. Finally, the faulty value $\hat{f}_{p 1}$ is estimated and it matches with the given value $f_{p 1}$, see Figure 4 .
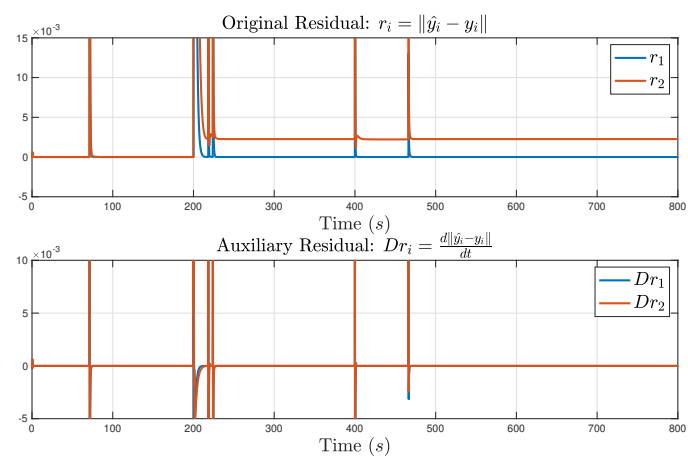

Fig. 3: Residuals: $h_{p}$ is faulty at $200 \mathrm{~s}$.

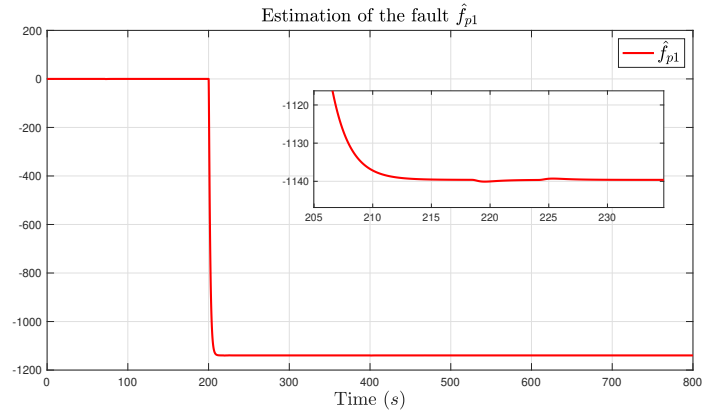

Fig. 4: Estimated fault value $\hat{f}_{p 1}$ when $h_{p}$ is faulty at $200 \mathrm{~s}$.

Finally, the fault information is used to redesign the controller and recover the fault. Figure 5 represents the process temperature $T_{p}$ when the AFTC system is applied and when it is not. We can see that, without the AFTC system, the process temperature $T_{p}$ cannot follow the desired value $T_{p, d}$. On the contrary, when the AFTC system is applied, the fault on $h_{p}$ is recovered, and the system continues to operate safely.
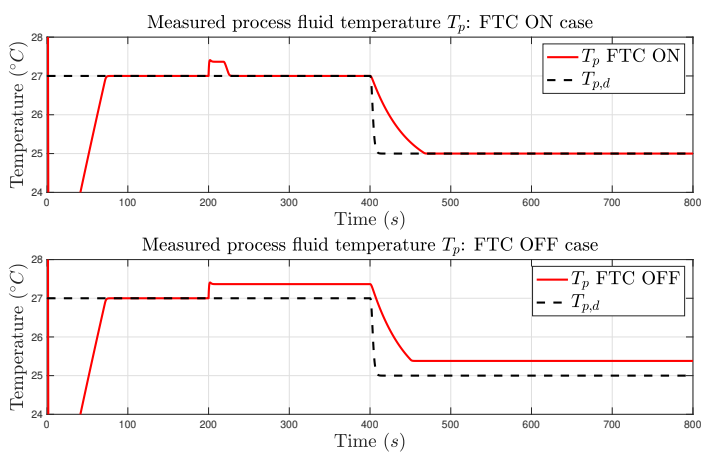

Fig. 5: Temperature $T_{p}$ under different cases: $h_{p}$ is faulty at $200 \mathrm{~s}$.

\section{B. Dynamic fault on $T_{p, \text { in }}$}

In the second case, a fault $f_{p 2}=-5{ }^{\circ} \mathrm{C}$ is added to the inlet temperature of process fluid $T_{p, i n}$ at $t_{f}=200 \mathrm{~s}$. Then, $T_{p, i n}^{f}=T_{p, i n}+f_{p 2}=73^{\circ} \mathrm{C}$. The residuals are represented in Figure 6. After $t_{f}=200 \mathrm{~s}$, and when the original residuals are stable, i.e. $D r_{i}=0, i=1,2$, the residual $r_{2}$ relative to $T_{p, i n}$, is at a zero value while the $r_{1}$ stays at a non zero value. Then, the fault is detected and isolated at the parameter $T_{p, i n}$. Finally the fault is estimated at $-5^{\circ} \mathrm{C}$, see Figure 7 .

Figure 8 represents the performance of the system when the AFTC system is applied and when it is not. We can see that the AFTC system provides fault recovery, so the system can operate safely.

\section{CONCLUSION}

In this paper, an adaptive observer-based AFTC system is used for an intensified HEX reactor with one unit and without taking into account a reaction. First, a control law based on a 

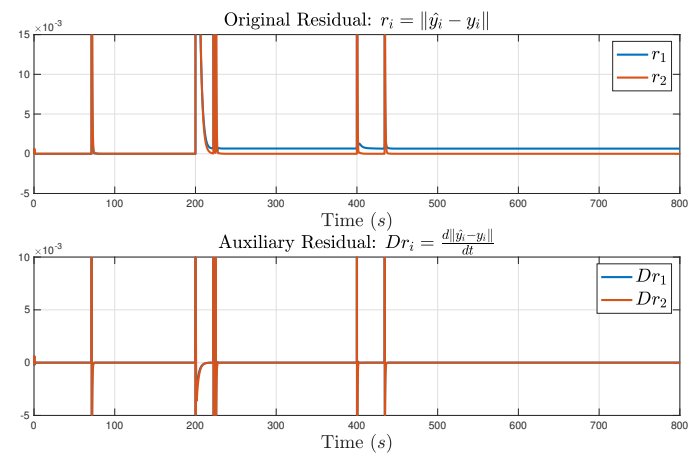

Fig. 6: Residuals: $T_{p, \text { in }}$ is faulty at $200 \mathrm{~s}$.

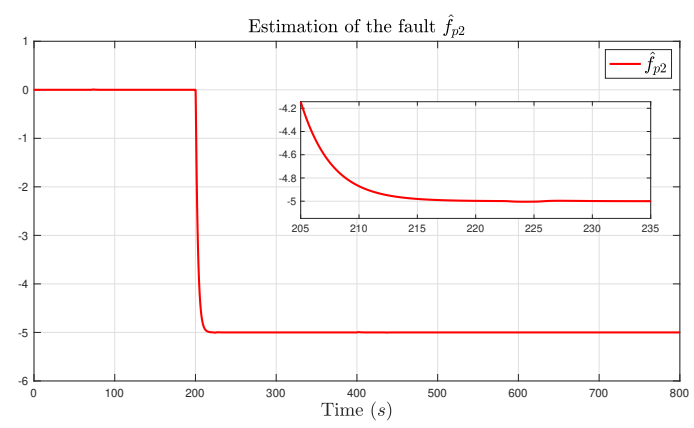

Fig. 7: Estimated fault value $\hat{f}_{p 2}$ when $T_{p, \text { in }}$ is faulty at $200 \mathrm{~s}$.
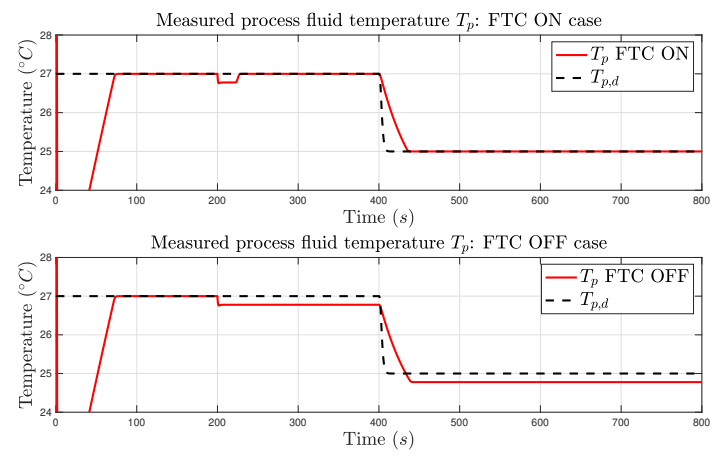

Fig. 8: Temperature $T_{p}$ under different cases: $T_{p, \text { in }}$ is faulty at $200 \mathrm{~s}$.

backstepping technique is designed. Then, a bank of adaptive observers is constructed to detect, isolate, and identify the dynamic fault. After that, the obtained fault information is used for the controller redesign procedure. The effectiveness of the adaptive observer-based AFTC method is validated by simulation on the HEX reactor system when a dynamic fault occurs. For future work, this AFTC system will be applied in order to recover the faults on the sensors and the actuators. Moreover, the whole system with the 17 units, as well as the measurement noise and parameter uncertainties will be taken into account.

\section{REFERENCES}

[1] Stankiewicz, Andrzej I., and Jacob A. Moulijn. "Process intensification: transforming chemical engineering." Chemical engineering progress 96, no. 1 (2000): 22-34.

[2] Etchells, J. C. "Process intensification: Safety pros and cons." Process Safety and Environmental Protection 83, no. 2 (2005): 85-89.

[3] Green, A., B. Johnson, and A. John. "Process intensification magnifies profits." Chemical engineering (New York, NY) 106, no. 13 (1999): 66-73.

[4] Theron, Félicie, Zoé Anxionnaz-Minvielle, Michel Cabassud, Christophe Gourdon, and Patrice Tochon. "Characterization of the performances of an innovative heat-exchanger/reactor." Chemical Engineering and Processing: Process Intensification 82 (2014): 30-41.

[5] Zhang, Youmin, and Jin Jiang. "Bibliographical review on reconfigurable fault-tolerant control systems." Annual reviews in control 32, no. 2 (2008): 229-252.

[6] Zhou, Kemin, John Comstock Doyle, and Keith Glover. Robust and optimal control. Vol. 40. New Jersey: Prentice hall, 1996.

[7] Noura, Hassan, Didier Theilliol, Jean-Christophe Ponsart, and Abbas Chamseddine. Fault-tolerant control systems: Design and practical applications. Springer Science \& Business Media, 2009.

[8] Zhang, Youmin, and Jin Jiang. "Bibliographical review on reconfigurable fault-tolerant control systems." Annual reviews in control 32, no. 2 (2008): 229-252.

[9] Amin, Arslan Ahmed, and Khalid Mahmood Hasan. "A review of fault tolerant control systems: advancements and applications." Measurement 143 (2019): 58-68.

[10] Ding, Steven X. "A survey of fault-tolerant networked control system design." IFAC Proceedings Volumes 45, no. 20 (2012): 874-885.

[11] Gertler, Janos J. "Survey of model-based failure detection and isolation in complex plants." IEEE Control systems magazine 8, no. 6 (1988): 3-11.

[12] Zhou, Yimin, Guoqing Xu, and Qi Zhang. "Overview of fault detection and identification for non-linear dynamic systems.” In 2014 IEEE International Conference on Information and Automation (ICIA), pp. 1040-1045. IEEE, 2014.

[13] Thirumarimurugan, M., N. Bagyalakshmi, and P. Paarkavi. "Comparison of fault detection and isolation methods: A review." In 2016 10th International Conference on Intelligent Systems and Control (ISCO), pp. 1-6. IEEE, 2016.

[14] He, Menglin, Zetao Li, Xue Han, Michel Cabassud, and Boutaib Dahhou. "Development of a Numerical Model for a Compact Intensified Heat-Exchanger/Reactor." Processes 7, no. 7 (2019): 454.

[15] He, Menglin, Zetao Li, Boutaib Dahhou, and Michel Cabassud. "The Fault Tolerant Control Design of an Intensified HeatExchanger/Reactor Using a Two-Layer, Multiple-Model Structure." Sensors 20, no. 17 (2020): 4888.

[16] Qian, Huaming, Yu Peng, and Mei Cui. "Adaptive observer-based fault-tolerant control design for uncertain systems." Mathematical Problems in Engineering 2015 (2015).

[17] Grouz, Faten, Lassaâd Sbita, Mohamed Boussak, and Amor Khlaief. "FDI based on an adaptive observer for current and speed sensors of PMSM drives." Simulation Modelling Practice and Theory 35 (2013): 34-49.

[18] Zhang, Ke, Bin Jiang, and Vincent Cocquempot. "Adaptive observerbased fast fault estimation." International Journal of Control, Automation, and Systems 6, no. 3 (2008): 320-326.

[19] Han, Xue, Zetao Li, Michel Cabassud, and Boutaib Dahhou. "A Comparison Study of Nonlinear State Observer Design: Application to an Intensified Heat-Exchanger/Reactor." In 2020 28th Mediterranean Conference on Control and Automation (MED), pp. 162-167. IEEE, 2020.

[20] Chen, Weitian, and Mehrdad Saif. An actuator fault isolation strategy for linear and nonlinear systems. Proceedings of the 2005, American Control Conference, 2005. IEEE, 2005.

[21] Fragkoulis, Dimitrios. Détection et localisation des défauts provenant des actionneurs et des capteurs: application sur un système non linéaire. Diss. Université de Toulouse, Université Toulouse III-Paul Sabatier, 2008. 